\title{
MEDIÇÃO EXPERIMENTAL E MODELAGEM TERMODINÂMICA DO PONTO DE NÉVOA DO BIODIESEL DE SEBO ANIMAL
}

\author{
C.P.G. de LIRA ${ }^{1}$, J. P. SILVA ${ }^{1}$, B. F. SANTOS ${ }^{1}$, A.S. GALINDO ${ }^{1}$, A.S.E.X. STRAGEVITCH ${ }^{1}$ \\ ${ }^{1}$ Universidade Federal de Pernambuco, Departamento de Engenharia Química \\ E-mail para contato: cinthyapetrucia@hotmail.com
}

\begin{abstract}
RESUMO - O ponto de névoa do biocombustível é a temperatura na qual ocorre o início de formação dos cristais parafínicos. Neste estudo, o ponto de névoa de amostras de biodiesel oriundo de sebo bovino foi determinado tanto por modelagem quanto experimentalmente, e seus valores foram comparados. Utilizou-se o modelo termodinâmico de equilíbrio sólido-líquido, baseado na igualdade de fugacidades das fases sólida e líquida para se encontrar o valor teórico através de simulação em MATLAB. Dados necessários para a modelagem foram obtidos por meio de análises cromatográficas e na literatura. O ponto de névoa experimental foi calculado em um Mini Pour/Cloud Point tester modelo MPC-101. O valor simulado médio encontrado foi 21,92 ${ }^{\circ} \mathrm{C}$ e o experimental foi $19,5{ }^{\circ} \mathrm{C}$, resultando num erro relativo de $11,04 \%$. As causas possíveis do erro foram a simplificação do modelo termodinâmico e o fato de aproximar o calor de fusão dos ésteres de formação do biodiesel para os de seus respectivos ácidos graxos.
\end{abstract}

\section{INTRODUÇÃO}

O biodiesel é um combustível composto de alquil ésteres de ácidos graxos de cadeia longa, derivados de óleos vegetais ou de gorduras animais (ANP, 2008). Apresenta diversas vantagens em relação ao combustível fóssil, como exemplo, combustão mais completa, redução das emissões de dióxido de carbono e enxofre, além de gerar emprego e renda especialmente nas fases de plantio, coleta e processamento dos grãos (CASTRO, 2009).

O uso dos resíduos gordurosos para fabricação do biodiesel colabora, ainda, para a redução de impactos ambientais, evitando o destino impróprio dos rejeitos que não são processados e descartados corretamente. Em média, a cada ano, são produzidos no Brasil 2,5 milhões de toneladas de gordura provenientes do abate industrial de aves, bovinos e suínos. O rendimento do processo de conversão dessas matérias-primas é de $65 \%$ a $70 \%$. Por exemplo, um quilo de rejeito animal pode gerar, em média, 650 mililitros de biocombustível (EMBRAPA, 2013).

Quando comparado ao biodiesel de óleos vegetais, o biodiesel obtido a partir da gordura animal apresenta algumas vantagens como: maior número de cetano, maior estabilidade à oxidação e menor 
índice de iodo. Mas o uso de gordura animal na preparação do biodiesel é prejudicado pela sua solidificação em temperatura ambiente, devido ao excesso de cadeias saturadas, aumentando o ponto de fusão do combustível, o que limita o seu uso em regiões de clima temperado e frio, já que o biodiesel solidificado ocasiona o entupimento de filtros e injetores em motores. (DOMINGOS et al.,2007).

Uma propriedade importante para se medir a qualidade do biodiesel no tocante à sua aplicabilidade em motores é o ponto de névoa, que é definido como a menor temperatura em que se observa a formação de uma turvação numa amostra do produto, indicando o início da sua cristalização, ou seja, sua solidificação (UFMG, 2013).

O presente trabalho objetiva predizer, através de uma modelagem termodinâmica, o ponto de névoa do biodiesel originado do sebo animal e em seguida, validar o algoritmo desenvolvido por meio de comparações com os dados experimentais obtidos.

\section{METODOLOGIA}

\subsection{Determinação do Ponto de névoa}

As análises referentes ao ponto de névoa tanto do biodiesel de gordura animal quanto dos seus sobrenadantes, foram realizadas no Laboratório de Energética Química da Unifacs - Salvador. O equipamento utilizado para realização das análises foi o Mini Pour/Cloud Point tester Model MPC101. O teste foi realizado de acordo com a norma ASTM D6749 - 02(2012).

O teste é realizado inicialmente com a preparação da cuba de amostra, preenchendo-a com o biodiesel e logo em seguida a mesma é inserida no bloco do banho. O sensor da unidade do banho é encaixado, para que os sensores de temperatura, fluidez e o tubo condutor de pressão não se encostem a cuba da amostra. Quando o feixe de luz detectar a formação de uma área turva, o equipamento irá fixar o valor da temperatura em que foi observado este fenômeno. Esta temperatura é o ponto de névoa.

O teste foi realizado para o biodiesel e seus respectivos sobrenadantes, para fins comparativos. Os sobrenadantes analisados foram obtidos colocando-se o biodiesel num frasco âmbar de $500 \mathrm{~mL}$ e posteriormente resfriadas até a separação de fases, onde se observava a formação de duas fases, uma sólida e outra líquida. A fração do biodiesel que não solidificava (sobrenadante) era retirada e colocada em outro frasco âmbar para uma nova refrigeração e posterior separação de fases. Esse procedimento foi realizado três vezes com a mesma amostra para que se obtivessem três sobrenadantes do biodiesel de composição distintas, as quais receberam os nomes de $1^{\circ}$ sobrenadante, $2^{\circ}$ sobrenadante e $3^{\circ}$ sobrenadante.

\subsection{Análise Cromatográfica}


Para o desenvolvimento do algoritmo, foram realizados ensaios cromatográficos com o intuito de obter a composição de ésteres do biodiesel de sebo e de seus respectivos sobrenadantes a fim de certificar se o precipitado formado possuía a mesma composição do biodiesel e calcular o equilíbrio sólido-líquido para obtenção do ponto de névoa através do software MATLAB.

A fração mássica de ésteres metílicos presentes na amostra foi determinada através de análise por cromatografia gasosa com detector de ionização de chama (GC-FID 17A Shimadzu). A análise permite a quantificação dos ésteres através de um procedimento de calibração interna; as condições cromatográficas utilizadas foram: temperatura da coluna de $200^{\circ} \mathrm{C}$ permanecendo por 17 minutos e temperatura do injetor de $250^{\circ} \mathrm{C}$ no modo split. A coluna DB-WAX é de $30 \mathrm{~mm}$ x $0,25 \mathrm{~mm}$ de sílica fundida com $0,25 \mu \mathrm{m}$ de filme líquido. O gás de arraste utilizado na coluna é hidrogênio. Razão de split 50:1.Pressão de $76 \mathrm{Kpa}$, velocidade linear de $38,7 \mathrm{~cm} / \mathrm{s}$ e tempo de análise de 17 minutos. A padronização interna foi com C17.

A determinação do percentual de cada éster nas misturas foi feita pelo método de normalização da área com fator de resposta (SCHULER, 2005). A seguinte relação foi usada, equação (1):

$$
C_{i}=A_{c i} / \sum A_{c i} \times 100
$$

Onde $\mathrm{A}_{\mathrm{ci}}$ é a área corrigida de um componente qualquer e é calculada com auxílio da equação (2):

$$
A_{c i}=A_{i} \cdot F_{i}
$$

$\mathrm{F}_{\mathrm{i}}$ é calculado experimentalmente a partir do cromatograma de uma mistura sintética (solução padrão) contendo todos os componentes da amostra real:

$$
F_{i}=C_{i} / A_{i}
$$

Onde $\mathrm{C}_{\mathrm{i}}$ é a concentração de um componente qualquer e $\mathrm{A}_{\mathrm{i}}$ sua respectiva área.

A tabela 2 apresenta as substâncias presentes nas amostras de biodiesel e as suas codificações. As frações mássicas de cada éster, encontradas por cromatografia, foram convertidas em frações molares, necessárias para as equações termodinâmicas de determinação do ponto de névoa.

Tabela 2 - Codificação dos ésteres das amostras de biodiesel.

\begin{tabular}{cc} 
Estér & Código \\
\hline Mirístato de metila & $\mathrm{C} 14: 0$ \\
Pentadecanoato de metila & $\mathrm{C} 15: 0$ \\
Palmitato de metila & $\mathrm{C} 16: 0$ \\
Palmitoleato de metila & $\mathrm{C} 16: 1$ \\
Estearato de metila & $\mathrm{C} 18: 0$ \\
Oleato de metila & $\mathrm{C} 18: 1 \mathrm{n}-9$ \\
Trans-11-octadecenoato de metila & $\mathrm{C} 18: 1 \mathrm{n}-7$ \\
Linoleato de metila & $\mathrm{C} 18: 2 \mathrm{n}-6$ \\
Linolênato de metila & $\mathrm{C} 18: 3 \mathrm{n}-3$
\end{tabular}


Araquidonato de metila

Behenato de metila
C20:0

$\mathrm{C} 22: 0$

\subsection{Simulação Numérica}

Para que a simulação em software seja possível, além das frações de ésteres nas amostras, são necessários alguns dados termodinâmicos (temperatura de fusão e calor de fusão) e as massas molares de cada éster presente na composição do biodiesel. Estes dados foram obtidos da literatura. Apenas para alguns ésteres, que não possuíam as entalpias de fusão disponíveis na literatura, utilizou-se o calor de fusão de seus ácidos graxos correspondentes, visto que, para a maioria dos ésteres, estes valores são suficientemente próximos (Imahara et al., 2006).

Uma forma teórica de se determinar o ponto de névoa é através de modelos termodinâmicos. Em temperaturas baixas, o biodiesel se separa em uma fase sólida e uma fase líquida. Quando estas fases entram em equilíbrio, além da igualdade de temperatura e pressão entre as fases, vale a igualdade das fugacidades de cada substância, dada pela equação 4.

$$
F_{i}^{l}=F_{i}^{s}(\forall i)
$$

Considerando os coeficientes de atividade e as frações molares das espécies em cada fase, a equação pode ser representada pela equação 5:

$$
x_{i}^{l} \gamma_{i}^{l} f_{i}^{l}=x_{i}^{s} \gamma_{i}^{s} f_{i}^{s}(\forall i)
$$

Onde $x \_i$ é a fração molar da espécie $i, \gamma \_i$ é o seu coeficiente de atividade, e f_i é a fugacidade da substância pura. Relacionando as fugacidades dos componentes puros com a variação na energia de Gibbs do sistema, a equação pode ser desenvolvida, resultando na equação 6 (Prausnitz et al., 1986):

$$
\ln \left(\frac{x_{i}^{g} y_{i}^{s}}{x_{i}^{2} y_{i}^{i}}\right)=\frac{\Delta H_{i, f u s}}{R T_{i, t r p}}\left(\frac{T_{i, f u s}}{T}-1\right)-\frac{\Delta C p_{i}}{R}\left(\frac{T_{i, f u s}}{T}-1\right)+\frac{\Delta C p_{i}}{R} \ln \frac{T_{i, f u s}}{T}(\forall i)
$$

Sendo a variação da capacidade calorífica entre as fases desprezível em face da variação da entalpia de fusão, admitindo solução líquida ideal $\left(\gamma_{-} \mathrm{i}^{\wedge} \mathrm{l}=1\right)$ e completa imiscibilidade para todas as espécies no estado sólido ( $\mathrm{x}_{-} \mathrm{i}^{\wedge} \mathrm{S} \gamma_{-} \mathrm{i}^{\wedge} \mathrm{S}=1$ ) (Smith et al., 2007), a equação se reduz para a equação 7:

$$
\ln x_{i}=\frac{\Delta H_{i, f u s}}{R T_{i, f u s}}\left(\frac{T_{i, f u s}}{T}-1\right)
$$

Esta equação pode ser usada para se determinar a menor temperatura em que uma fase líquida de composição conhecida entre em equilíbrio com sua fase sólida, ou seja, o início da cristalização. 
Ao se rearranjar a equação 7 , obtemos:

$$
T=\frac{T_{\text {i,fus }}}{\left(1+\frac{R T_{\text {i,fus }} \ln \left(x_{i}\right)}{\left.\Delta H_{i, f u s}\right)}\right.}(\forall i)
$$

Conhecendo-se o calor de fusão e a temperatura de fusão de cada substância, além da composição da fase líquida, a equação 8 pode ser resolvida para se determinar o ponto de névoa. Com base nos dados da fração de ésteres, temperaturas de fusão e calor de fusão, esta equação foi utilizada no desenvolvimento do algoritmo mostrado no Apêndice A.

O algoritmo desenvolvido para obter o ponto de névoa fornece a temperatura inicial de cristalização de cada éster presente no biodiesel.

A partir da maior temperatura obtém-se o ponto de névoa, já que a formação dos cristais começam a partir do éster que apresenta a maior temperatura de cristalização. Com isso, a maior temperatura encontrada em cada amostra será considerada o ponto de névoa, pois a partir da mesma começa a ocorrer a formação dos cristais.

\section{RESULTADOS E DISCUSSÃO}

A partir dos dados obtidos na literatura e das análises cromatográficas, simulou o ponto de névoa por meio do software MATLAB. Os valores obtidos encontram-se na Tabela 3

Tabela 3 - Temperaturas dos ésteres obtidas através do MATLAB.

\begin{tabular}{|c|c|c|c|c|}
\hline \multirow{2}{*}{$\begin{array}{c}\text { Éster de ácido } \\
\text { graxo }\end{array}$} & \multicolumn{4}{|c|}{ Temperatura $\left({ }^{\circ} \mathrm{C}\right)$} \\
\cline { 2 - 5 } & $\begin{array}{c}\text { Biodiesel de } \\
\text { sebo }\end{array}$ & $1^{\circ}$ sobrenadante & $2^{\circ}$ sobrenadante & $\begin{array}{c}3^{\circ} \\
\text { sobrenadante }\end{array}$ \\
\hline C $14: 0$ & -23 & -23 & -26 & -23 \\
C15:0 & -53 & -53 & -55 & -53 \\
C 16:0 & 14 & 15 & 12 & 15 \\
C $16: 1$ & -47 & -47 & -48 & -47 \\
C 18:0 & 22 & 22 & 20 & 22 \\
C 18:1 n-9 & -35 & -35 & -35 & -35 \\
C 18:2 n-6 & -61 & -62 & -53 & -62 \\
C 18:3 n-3 & -89 & -89 & -81 & -89 \\
C18:1 n-7 & -41 & -45 & -53 & - \\
C 20:0 & -17 & -17 & -10 & - \\
C 22:0 & 12 & - & - & \\
\hline
\end{tabular}

As temperaturas destacadas correspondem as maiores temperaturas para cada amostra analisada. A partir da literatura observa-se que os percentuais mássicos do C18:0 e o C16:0 apresentam maior composição dentre os ésteres saturados. Sendo o ponto de fusão de ésteres 
saturados maiores ao de insaturados. Era de esperar que o ponto de névoa fosse determinado por um éster de cadeia saturada.

Por meio de dados da literatura notou-se que tanto o calor de fusão quanto a temperatura de fusão do C18:0 são superiores ao do C16:0, sendo assim o C18:0 foi o éster que determinou a temperatura de início de cristalização do biodiesel.Comprovando que quanto maior a presença de ésteres saturados, maior a temperatura de cristalização em regiões mais frias.

Os pontos de névoa e fluidez do biodiesel obtidos experimentalmente de sebo e de seus sobrenadantes estão apresentados na Tabela 4 para que possam ser comparados.

Tabela 4 - Pontos de névoa e fluidez para o biodiesel e seus respectivos sobrenadantes.

\begin{tabular}{c|c} 
Biodiesel de sebo & 20 \\
$1^{\circ}$ sobrenadante & 20 \\
$2^{\circ}$ sobrenadante & 19 \\
$3^{\circ}$ sobrenadante & 19
\end{tabular}

Os valores das temperaturas do ponto de Névoa obtidos através do algoritmo desenvolvido em MATLAB encontram-se na Tabela 5.

Tabela 5 - Temperaturas do ponto de névoa obtidas através do MATLAB.

\begin{tabular}{c|c} 
Biodiesel de sebo & 22,34 \\
$1^{\circ}$ sobrenadante & 22,45 \\
$2^{\circ}$ sobrenadante & 20,43 \\
$3^{\circ}$ sobrenadante & 22,46
\end{tabular}

Comparando os valores obtidos por simulação com os valores encontrados experimentalmente notamos que os mesmos estão próximos, apresentando um erro aproximadamente de $2^{\circ} \mathrm{C}$. A essa diferença atribui-se a aproximação feita para os valores do calor de fusão de ésteres metílicos que não foram encontrados na literatura com os dos seus respectivos ácidos graxos, bem como as considerações feitas na obtenção da fórmula de equilíbrio sólido-líquido. A Tabela 6 apresenta os erros relativos e absolutos entre os pontos de névoa experimental e calculado.

Tabela 6- Comparação entre valor simulado e valor experimental do ponto de névoa.

\begin{tabular}{c|c|c|c|c}
\hline Amostra & $\begin{array}{c}\text { Ponto de névoa } \\
\text { simulado }\left({ }^{\circ} \mathrm{C}\right)\end{array}$ & $\begin{array}{c}\text { Ponto de névoa } \\
\text { experimental }\left({ }^{\circ} \mathrm{C}\right)\end{array}$ & $\begin{array}{c}\text { Erro absoluto } \\
\left({ }^{\circ} \mathrm{C}\right)\end{array}$ & $\begin{array}{c}\text { Erro relativo } \\
(\%)\end{array}$ \\
\hline Biodiesel de sebo & 22,34 & 20,00 & 2,34 & 10,47 \\
$1^{\circ}$ sobrenadante & 22,45 & 20,00 & 2,45 & 10,91 \\
$2^{\mathbf{o}}$ sobrenadante & 20,43 & 19,00 & 1,43 & 7,00 \\
$3^{\text {o }}$ sobrenadante & 22,46 & 19,00 & 3,46 & 15,41 \\
Média & 21,92 & 19,50 & 2,42 & 11,04 \\
\end{tabular}




\section{CONCLUSÕES}

Tendo como objetivo a modelagem termodinâmica para predição do ponto de Névoa do biodiesel, o trabalho desenvolvido apresentou-se coerente e o algoritmo previu satisfatoriamente o início da fusão do biodiesel e seus sobrenadantes.

O resultado experimental foi comparado com o modelo teórico, obtido através de equações termodinâmicas simplificadas e do uso de software para resolução das mesmas. O erro relativo foi de $11,04 \%$, dentro dos padrões esperados, já que muitas aproximações foram feitas, especialmente ao se utilizar o calor de fusão dos ácidos graxos encontrados na literatura, para os casos em que esta não possuía entalpias de fusão disponíveis.

\section{REFERÊNCIAS}

ANP - AGÊNCIA NACIONAL DO PETRÓLEO, GÁS NATURAL E BIOCOMBUSTÍVEIS; Resolução Nº 7 de 19/3/2008; Diário Oficial da União de 20/3/2008.

ASTM D6749-02(2012) Standard Test Method for Pour Point of Petroleum Products (Automatic Air Pressure Method)

Castro, B.C.S. Otimização das Condições da Reação de Transesterificação e Caracterização dos Rejeitos dos Óleos de Fritura e de Peixe para Obtenção de Biodiesel. Dissertação de Mestrado, UFRJ, 2009.

Domingos, A. K.; Vecchiatto, W.; Wilhelm, H. M.; Ramos, L. P.; J. Braz. Chem. Soc. 2007, 18, 416.

Imahara H., Minami E., Saka S.; Thermodynamic study on cloud point of biodiesel with its fatty acid composition. Fuel, Vol. 85, No. 12-13. (September 2006), pp. 1666-1670

Prausnitz, J. M.; Lichtenthaler, R. N.; Azevedo, E. G. A. Molecular thermodynamics of Fluid-phase equilibria. 2 ed. New Jersey, Prentice-Hall, 1986.

Schuler, A. CROMATOGRAFIA A GÁS E A LÍQUIDO. Detetores, aquisição de dados, validação e avaliação estatística. $9^{\circ}$ Edição, 2005. 77p

UFMG - UNIVERSIDADE FEDERAL DE MINAS GERAIS. Departamento de Engenharia Mecânica. Supervisionada por Paulo Cesar da Costa Pinheiro. Apresenta parte integrante do seminário de combustíveis. Disponível em: <http://www.demec.ufmg.br/disciplinas/ema003/liquidos/diesel/especifi.htm>. Acesso em: Junho de 2013

EMBRAPA,2013. Disponível em:<http://www.agencia.cnptia.embrapa.br/gestor/agroenergia/arvore/ 
CONT000fj1om7kf02wyiv802hvm3jholyoom.html>Acesso em: 11 mar. 2013

\section{APÊNDICE A}

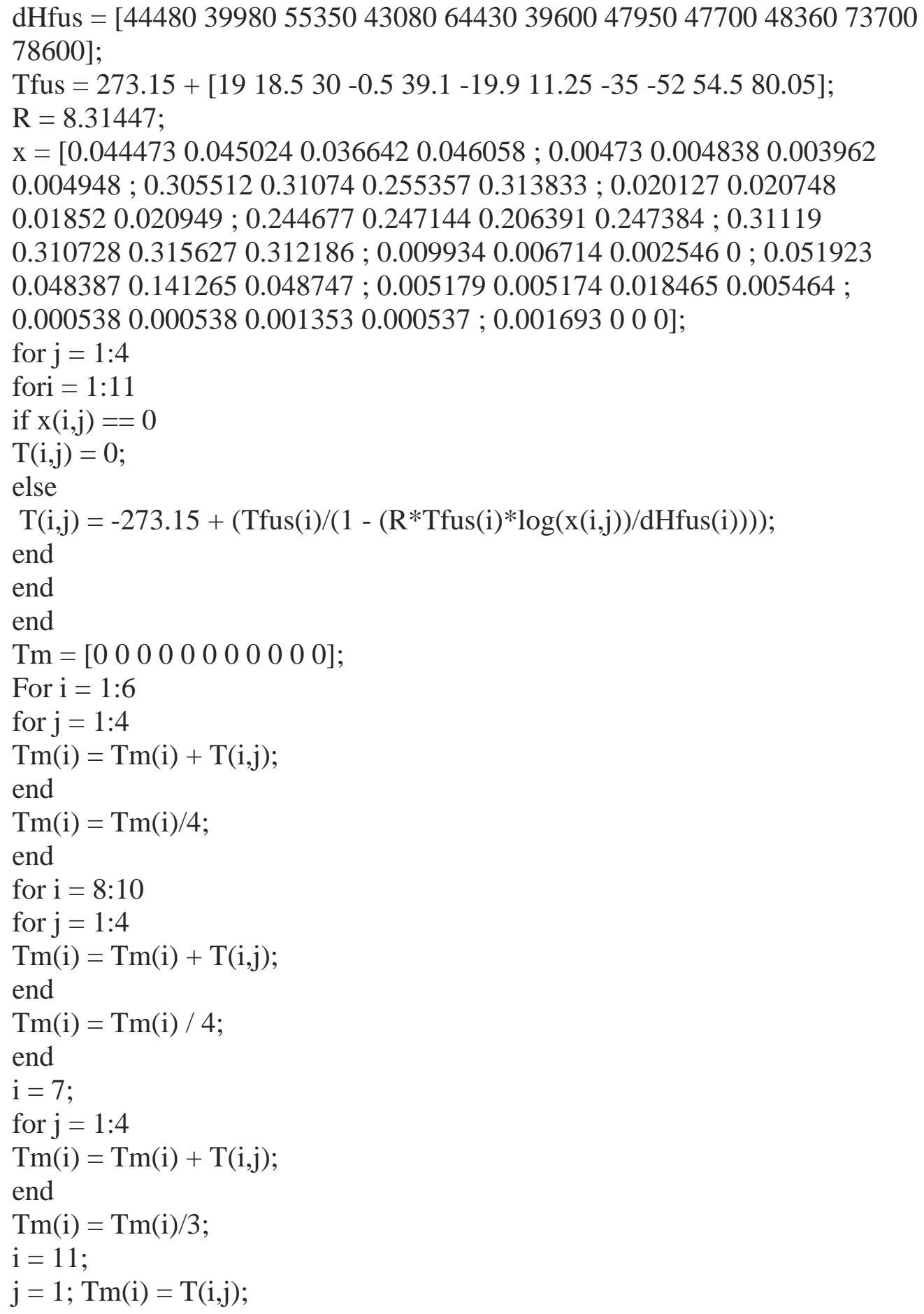

